\section{Patrones de consumo y genotipos determinantes de la tolerancia al alcohol: estudio en estudiantes universitarios de Santiago de Chile}

\author{
CONSTANZA P. SILVA ${ }^{1, a}$, SERGIO V. FLORES ${ }^{1, b}$
}

\section{Alcohol consumption patterns and genotypes determining alcohol tolerance in Chilean university students}

Background: Alleles involved in inefficient $\left(A D H 1 B 2^{\star} 2\right.$ and $\left.A L D H 22^{*} 2\right)$ or efficient (SNP6, ADH4 gene) alcohol metabolism may influence the risk of alcoholism. Alcoholism susceptibility has been classified as protector and risk-dependence phenotypes, associated with inefficient and efficient alcohol genetic metabolizing variants, respectively. Aim: To investigate the possible association between genetic protective and risk-dependence variants and alcohol intake patterns. Material and Methods: Saliva DNA samples were obtained and the AUDIT (Alcohol Use Disorders Identification Test) questionnaire was applied to 210 university students aged between 18 and 25 years old. Results: No statistically significant association between protective or risk-dependence genetic variants and alcohol pattern intake was detected. However, new categories of alcohol intake patterns-not included in the AUDIT questionnaire-were identified. Conclusions: No association between the protector and risk-dependence phenotypes and patterns of alcohol consumption was detected in this sample of students.

(Rev Med Chile 2015; 143: 707-715)

Key words: Alcohol Dehydrogenase; Alcohol Drinking; Aldehyde Dehydrogenase; Genetics, Population; Genetic Variation.

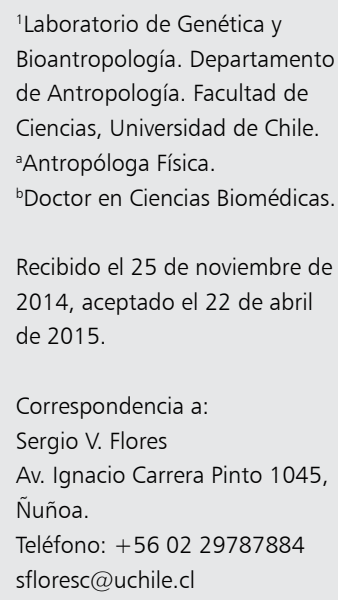

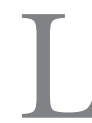
a metabolización del etanol es realizada por dos sistemas enzimáticos en el hígado: enzima Alcohol Deshidrogenasa (ADH) y Aldehído Deshidrogenasa (ALDH), codificadas en humanos por una familia de genes ADH en el cromosoma 4 y ALDH en el cromosoma 12. Además de presentar variabilidad genética intra e interpoblacional, los genotipos definen variabilidad en la respuesta fisiológica al consumo de alcohol. Se ha propuesto que estos mismos genes participan en la vulnerabilidad a la dependencia al alcohol (DA), asociándose a "fenotipos protectores" o de "riesgo-dependencia", 2 .

Tanto el polimorfismo $A D H 1 B^{\star} 2$ (rs1229984) del gen $A D H 1 B$ como $A L D H 2^{\star} 2$ (rs671) del gen $A L D H 2$ presentan alta frecuencia en poblaciones del Este de Asia, llegando a valores cercanos a la fijación ${ }^{3-6} . A D H 1 B^{*} 2$ posee una tasa metabólica elevada en relación a otros alelos del gen $A D H 1 B$ (e.g. $\left.A D H 1 B^{*} 1\right)^{1}$, lo que determina la acumulación de sustancias tóxicas en el organismo propias de la metabolización del etanol (e.g. acetaldehído) y por ende reacciones iniciales de intoxicación tras la ingesta. Por otro lado, el alelo $A L D H 2 * 2$ codifica una enzima inactiva, generando una respuesta llamada "síndrome asiático de rubor facial" . En ambos casos estas son conocidas como variantes de "protección" contra el alcoholismo. 
El alelo de riesgo-dependencia al alcohol más relevante ha sido asociado con el gen de $A D H 4$ y corresponde al SNP6 (rs1800759). Presenta una alta frecuencia en población europea y amerindia de norteamérica (SW American indian tribe) y casi inexistente en el Este de Asia. La presencia de la enzima ADH4 determina metabolización eficiente del etanol en episodios de intoxicación, asociándose así a fenotipo de "riesgo", ya que permitiría un mayor consumo de alcohol a largo plazo ${ }^{4,5}$.

La DA o alcoholismo es una enfermedad compleja y multifactorial debido a la interacción de factores ambientales y genéticos ${ }^{1,8-12}$. La OMS publicó un estudio sobre los patrones de consumo de alcohol en adultos, donde se destaca el alto consumo y patrones riesgosos tanto para Europa como América; por el contrario, destaca el bajo nivel que se presenta en $\mathrm{Asia}^{13}$. En Latinoamérica, Chile es el país con la mayor prevalencia de consu$\mathrm{mo}^{14}$, siendo el país con mayor cantidad de litros per cápita promedio (6,6 litros), tanto en hombres (13,9 litros) como mujeres (5,5 litros) ${ }^{15}$.

La necesidad de sistematización en la implementación de políticas públicas en torno al consumo de alcohol ha impulsado el diseño de instrumentos para la evaluación de los patrones de consumo. El cuestionario AUDIT (Alcohol Use Disorders Identification Test), tiene el objetivo de detectar tempranamente a personas con problemas de dependencia. Permite una aplicación rápi$\mathrm{da}, \sin$ costo y no requiere mayor entrenamiento ${ }^{16}$. La primera edición de este manual fue publicada en 1989 (Documento No WHO/MNH/89.4) y fue actualizada en 1992 (WHO/PSA/92.4). Esta encuesta presenta de 0 a 40 puntos, permitiendo clasificar a los individuos según tres categorías; consumo de riesgo, perjudicial y dependencia. El cuestionario ha sido validado para diferentes poblaciones, países y contextos culturales -Chile incluido- constituyéndose un instrumento útil y cada vez más aplicado. Se ha sugerido que los puntajes de corte para la clasificación de patrones de consumo de alcohol se ajusten al contexto de riesgo de cada país (Tabla 1$)^{16,17}$.

El estudio, análisis e interpretación en torno al consumo y riesgo-dependencia al alcohol en población chilena ha sido abordado principalmente desde la perspectiva del detrimento de la salud, el riesgo social y estereotipos asociados ${ }^{14,18}$. No obstante, es necesaria la indagación del trasfondo genético (herencia) y factores socioculturales (am- bientales) que influyen en estos fenotipos en la población chilena. Estas variables son indispensables para evaluar la diversidad y distribución poblacional de los patrones de consumo y cómo afectan al diseño de políticas públicas de prevención. En este estudio analizamos la asociación entre las variantes de protección y riesgo involucradas en la metabolización del alcohol y los patrones de consumo de esta sustancia en un grupo de estudiantes universitarios de Santiago de Chile.

\section{Materiales y Método}

\section{Sujetos}

Consistió en 210 estudiantes de pregrado de la Universidad de Chile entre 18 y 25 años, no emparentados, de ambos sexos y residentes en el Área Metropolitana del Gran Santiago. Se recolectaron $2 \mathrm{ml}$ de saliva para análisis genético y se aplicó el cuestionario AUDIT a cada participante. El tamaño de la muestra fue estimado para una población de 6 millones de habitantes, con 95\% de confianza y un intervalo de confianza de \pm 7 .

Se aseguró la confidencialidad mediante la utilización de códigos de identificación tanto para muestras de saliva como para los cuestionarios. Esto fue expuesto a los participantes mediante un consentimiento informado previamente aprobado por el Comité de Ética de la Investigación en Ciencias Sociales y Humanidades de la Facultad de Filosofía y Humanidades de la Universidad de Chile.

\section{Métodos}

a) Encuesta AUDIT

Para la estandarización de la aplicación del cuestionario AUDIT en esta investigación, se tomaron las siguientes medidas: (i) respecto a la cantidad de alcohol por cada trago se hizo referen-

\section{Tabla 1. Rangos para clasificación de patrones de consumo según puntaje AUDIT, propuesto por la OMS y sugerido para población chilena ${ }^{14}$}

\begin{tabular}{|c|c|c|}
\hline $\begin{array}{l}\text { Categorías de } \\
\text { consumo }\end{array}$ & OMS & Chile \\
\hline Consumo de riesgo & 8 a 15 puntos & 6 a 8 puntos \\
\hline Consumo prejudicial & 16 a 19 puntos & Sobre 9 puntos \\
\hline Dependencia & Sobre 20 puntos & \\
\hline
\end{tabular}


cia al contenido promedio de una lata de cerveza en Chile y un trago típico fue definido como aquel que contiene $13 \mathrm{~g}$ de alcohol, equivalente a un vaso; (ii) se incorporó la categoría de "consumo sin riesgo" para individuos con puntajes por debajo de los utilizados para su clasificación según la OMS y la escala propuesta para Chile; (iii) individuos con puntaje AUDIT igual a cero fueron clasificados como "no consumidores de alcohol".

\section{b) Genotipificación}

La extracción de ADN de las muestras de saliva fue llevada a cabo a partir de un protocolo ad $h o c^{19}$ en el Laboratorio de Antropología Genética y Bioantropología, de la Facultad de Ciencias Sociales de la Universidad de Chile.

El análisis genético comprendió tres etapas: (i) Búsqueda de alelos protectores y de riesgo-dependencia en bases de datos genómicas ${ }^{20}$ para el diseño de partidores de $\mathrm{PCR}^{20,21}$ y búsqueda de sitios de corte con enzimas de restricción ${ }^{22,23}$; (ii) Estandarización de protocolos para PCR (Polymerase Chain Reaction) y RFLP (Restriction Fragment Length Polymorphism); (iii) genotipificación de variantes protectoras y de riesgo-dependencia; $y$ (iv) Análisis genético-poblacional.

Los genes y alelos analizados en este estudio son aquellos asociados a función enzimática $\left(A D H 1 B^{\star} 2\right.$ y $\left.A L D H 2^{\star} 2\right)$ y a su asociación a la dependencia del alcohol (ADH4: SNP6 $)^{4,5}$.

Un conjunto de productos de PCR $(\mathrm{n}=20)$ fueron secuenciados (Macrogen, Korea), ratifi- cando la amplificación del fragmento génico de interés y el protocolo de RFLP.

\section{c) Análisis estadístico}

Se realizaron en tres etapas: (i) encuesta AUDIT y patrones de consumo; (ii) variabilidad genética; y (iii) asociación entre patrones de consumo y genotipos. La normalidad de puntajes AUDIT se evaluó con la prueba de Shapiro-Wilk. Los softwares utilizados en estos análisis corresponden a R Core Team, R Studio, ggPlot y Arlequin 3.5.1.3..$^{24-27}$.

\section{Resultados}

\section{a) Análisis encuesta AUDIT}

Se clasificó a los individuos según las categorías AUDIT propuestas tanto por la OMS como por las recomendadas para Chile. Se observó que existen frecuencias disímiles respecto al "consumo de riesgo", "perjudicial" y "dependencia" al alcohol, entre la escala propuesta por la OMS y la recomendada para Chile (Figura 1a y Figura 1b).

Respecto al promedio del puntaje AUDIT entre hombres $(6,67 \pm 4,59)$ y mujeres $(5,92 \pm$ $4,72)$ no se encontraron diferencias significativas (Mann-Withney, $\mathrm{w}=4630,5, \mathrm{p}_{\mathrm{m}-\mathrm{w}}=0,2012$ ) (Figura 2), así como tampoco para la proporción de individuos por patrón de consumo en ambos grupos (test de $\chi^{2}=0,9592, \mathrm{p}_{\mathrm{ji}}{ }^{2}=0,811$ ).

En el Análisis de Correspondencia Múltiple

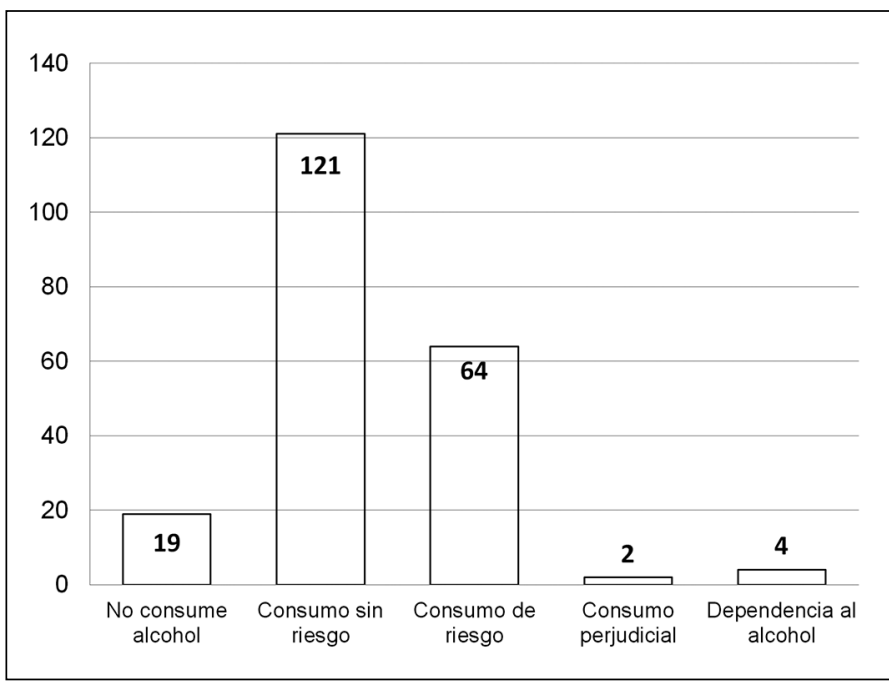

Figura 1a. Categorías patrones de consumo OMS. Distribución de los 210 individuos encuestados y clasificados en patrones de consumo según los puntajes de corte propuestos por la OMS. En su mayoría los individuos se encuentran en la categoría de "consumo sin riesgo", seguido por aquellos que se encuentran en la categoría de "consumo de riesgo". 

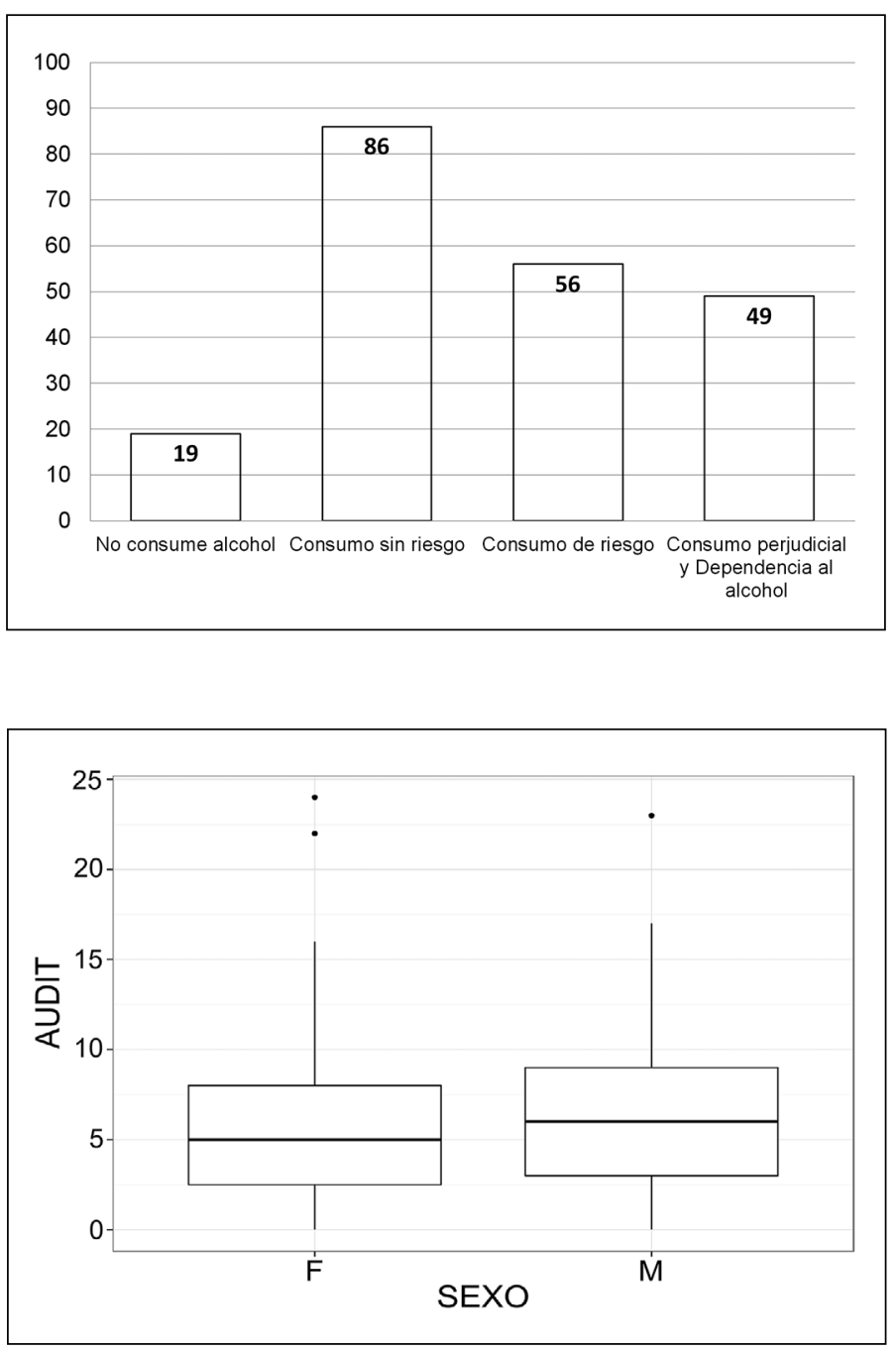

Figura 1b. Categorías patrones de consumo Chile. Distribución de los 210 individuos encuestados y clasificados en patrones de consumo según los puntajes de corte propuestos para población Chilena. En su mayoría los individuos se encuentran en la categoría de "consumo sin riesgo", seguido por aquellos que se encuentran en la categoría de "consumo de riesgo". No obstante, a diferencia de la Figura 1a, la distribución es más homogénea, encontrando mayor cantidad de individuos en la categoría de "consumo perjudicial y dependencia".
Figura 2. Patrones de consumo y genotipos determinantes de la tolerancia al alcohol: estudio en estudiantes universitarios de Santiago de Chile. Puntajes AUDIT según sexo. Diagrama de caja del puntaje AUDIT del grupo femenino (F) y masculino (M). Se muestra la dispersión y mediana para ambos grupos.
(MCA) los dos primeros ejes explicaron $21 \%$ de la varianza total $(\operatorname{Dim} 1=13,09 \%$ y $\operatorname{Dim} 2=8,75 \%)$. Las variables más explicativas correspondieron a los métodos de categorías AUDIT (OMS y Chile) y el puntaje AUDIT per se (Figura 3). Las variables menos explicativas fueron la edad y el sexo.

$\mathrm{Al}$ analizar la distribución de los individuos según su puntaje AUDIT, se observaron grupos discretos de consumo de alcohol (Figura 4), ubicándose los individuos de menor puntaje en el cuadrante II, mientras que los de mayor en el cuadrante I y IV. Un tercer análisis con las respuestas de cada uno de éstos al cuestionario develó que "subpatrones" de consumo de alcohol en los individuos de alto puntaje, ubicados en los cuadrantes I y IV. En el cuadrante I se ubican individuos clasificados con "episodios alcohólicos" y que presentan mayor frecuencia de consecuencias relacionadas con el consumo (pérdida de memoria, beber en ayunas, sentimientos de culpa, personas heridas, etc.). En el cuadrante IV se ubican individuos con alto y sostenido consumo, con menor frecuencia de consecuencias relacionadas con el hábito.

\section{b) Análisis variantes genéticas}

Para el marcador $A D H 1 B^{\star} 2$ fueron analizados 410 alelos $^{1}$ (Los individuos restantes presentaron problema en la amplificación en el PCR). La frecuencia del alelo "G" alcanzó 5,6\% (Tabla 2); 

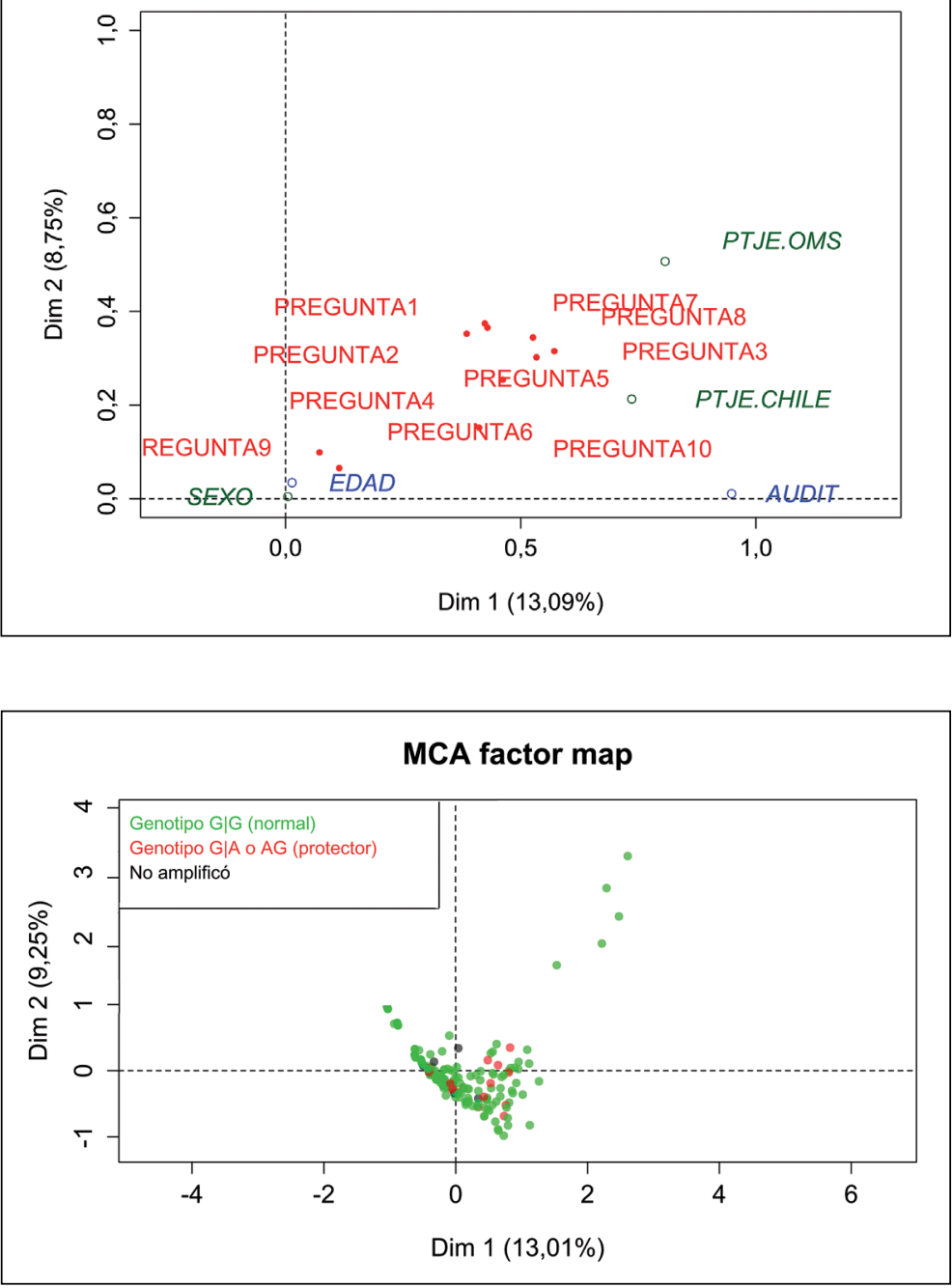

Figura 3. Análisis de Correspondencia Múltiple (MCA). Análisis de Correspondencia Múltiple (MCA). En color rojo se encuentran las preguntas de la encuesta AUDIT, en verde las variables suplementarias categóricas y en azul las variables suplementarias continuas. Las dimensiones explican cerca del $21 \%$ de la varianza total de la muestra. Se observan dos grupos de preguntas (pregunta 6,9 y pregunta $1,2,3,4,5,7,8,10$ ). Las variables menos explicativas corresponden a sexo y edad. Por otro lado, las variables de categorización de la OMS y Chile recogen mayor variabilidad que el puntaje AUDIT.

Figura 4. MCA distribución individuos según puntaje AUDIT y patrones de consumo. MCA de dispersión de los individuos según puntaje AUDIT. Como variable suplementaria fue sobrepuesta la clasificación de patrones de consumo (puntaje sugerido para Chile) a cada uno de los individuos. Se observan claros grupos de consumo de alcohol. Los individuos que no consumen alcohol y de bajo consumo se ubican en el sector izquierdo del gráfico y hacia el centro; mientras que los de mayor puntaje $\mathrm{AU}$ DIT se ubican en el cuadrante I (puntajes 16 a 24 puntos) y IV (puntajes 15 a 17).

Tabla 2. Porcentajes en la muestra de alelos, genotipo y fenotipo de cada marcador genético

\begin{tabular}{|c|c|c|c|c|c|}
\hline Alelos & Porcentajes & Genotipo & Porcentajes & Fenotipo & Porcentajes \\
\hline \multicolumn{6}{|c|}{$A D H 1 B * 2(\mathrm{rs} 1229984)$} \\
\hline $\mathrm{G}$ & $94,3 \%(n=387)$ & $\mathrm{G} \mid \mathrm{G}$ & $88,7 \%(n=182)$ & Normal (G|G) & $88,7 \%(n=182)$ \\
\hline \multirow[t]{2}{*}{ A } & $5,6 \%(n=23)$ & $\mathrm{G} \mid \mathrm{A}$ & $11,2 \%(n=23)$ & Riesgo reducido $(G \mid A)$ & $11,2 \%(n=23)$ \\
\hline & & $A \mid A$ & $0 \% \quad(n=0)$ & $\operatorname{Protector}(\mathrm{A} \mid \mathrm{A})$ & $0 \% \quad(n=0)$ \\
\hline \multicolumn{6}{|c|}{$A L D H 2 * 2(\mathrm{rs} 671)$} \\
\hline G & $100 \%(n=61)$ & $\mathrm{G} \mid \mathrm{G}$ & $100 \%(n=61)$ & Normal (G G) & $100 \%(n=61)$ \\
\hline \multirow[t]{2}{*}{ A } & $0 \%(n=0)$ & $\mathrm{G} \mid \mathrm{A}$ & $0 \%(n=0)$ & Protector (A|A y $G \mid A)$ & $0 \%(n=0)$ \\
\hline & & $\mathrm{A} \mid \mathrm{A}$ & $0 \%(n=0)$ & & \\
\hline \multicolumn{6}{|c|}{ ADH4 (rs1800759) } \\
\hline $\mathrm{G}$ & $33,9 \%(n=140)$ & $\mathrm{G} \mid \mathrm{G}$ & $7,2 \%(n=15)$ & Normal (G|G y G $\mid T$ ) & $60,5 \%(n=125)$ \\
\hline \multirow[t]{2}{*}{$T$} & $66,0 \%(n=272)$ & $T \mid G$ & $33,3 \%(n=110)$ & Riesgoso $(\mathrm{T} \mid \mathrm{T})$ & $39,3 \%(n=81)$ \\
\hline & & $T \mid T$ & $39,3 \%(n=81)$ & & \\
\hline
\end{tabular}


porcentajes similares a los muestreados en otras regiones de $\mathrm{América}^{2}\left(\chi^{2}=1,7572, \mathrm{gl}=1\right.$, $\left.\mathrm{p}_{\mathrm{x} 2}=0,185\right)$ por el proyecto 1000Genomes (25). El valor de Fst fue de 0,00300.

El marcador $A L D H 2^{\star} 2$ se descartó como un marcador informativo ya que el resultado fue de $100 \%$ para el alelo normal G. La ausencia de alelo ' $A$ ' es consistente con el muestreo para América realizado por el proyecto 1000 Genomes.

Para el marcador ADH4 SNP6 fueron analizados 412 alelos $^{1}$. La frecuencia del alelo de riesgo "T" para la muestra alcanzó $66 \%$, porcentajes similares a los de otras regiones de América (Población correspondiente a Medellín, Colombia, ancestría mexicana en Los Ángeles, Estados Unidos de Norteamérica y a Puerto Rico) (Test de
Fisher, $\left.\mathrm{p}_{\mathrm{F}}=0,288 ; \mathrm{Fst}=0,02123\right)$. Las diferencias fueron sólo significativas con Puerto Rico (Test exacto de Fisher, $\mathrm{p}=0,0013$ ), con un valor de Fst $=0,005337$, reflejando así la diferenciación genética de mayor magnitud entre estas poblaciones.

Todos los marcadores se encontraron en equilibrio de Hardy-Weinberg. Los genotipos y fenotipos no presentaron diferencias significativas con la muestra de América.

\section{c) Análisis asociación entre patrones de consumo y genotipos}

Se realizó un segundo MCA para indagar de forma exploratoria si el factor genético podría estar asociado a la distribución según puntaje AUDIT de los individuos. De esta manera, al MCA
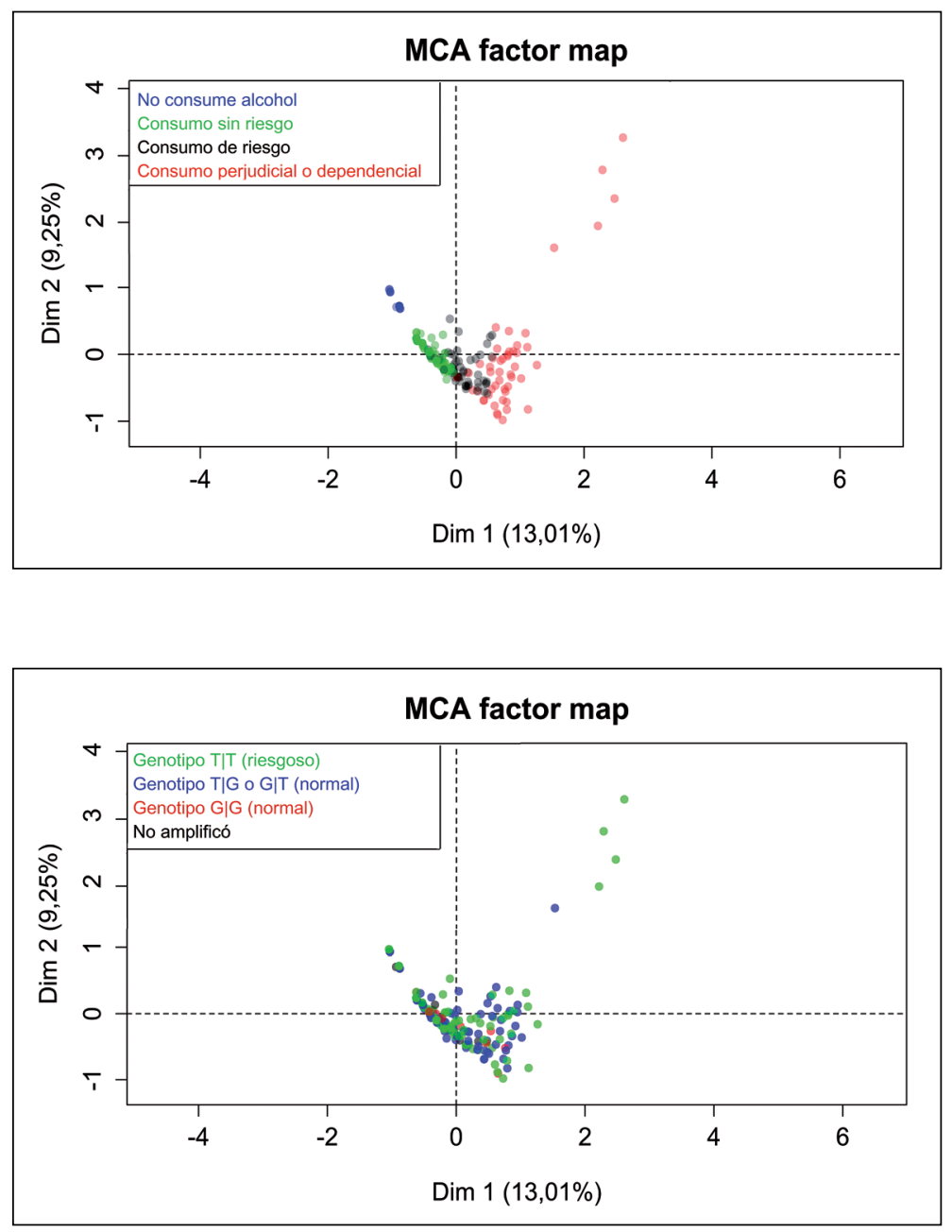

Figura 5. MCA distribución de individuos según puntaje AUDIT y patrón de consumo de alcohol y genotipos para el marcador $A D H 1 B$. Dispersión de los individuos respecto de la encuesta AUDIT. Como variable suplementaria fue sobrepuesto el genotipo para el marcador $A D H 1 B$. Existe baja representación del genotipo protector $(G \mid A)$ en la muestra. Se destaca que individuos en la categoría de consumo prejudicial y dependencia al alcohol presentan en su mayoría fenotipo normal $(G \mid G)$.
Figura 6. MCA distribución de individuos según puntaje AUDIT y patrón de consumo de alcohol y genotipos para el marcador $A D H 4$. Dispersión de los individuos respecto de la encuesta AUDIT. Como variable suplementaria fue sobrepuesto el genotipo para el marcador ADH4 (SNP6). Es posible observar que no existe relación entre los genotipos y patrones de consumo. En su mayoría aquellos individuos con mayor consumo de alcohol presentan un genotipo normal (G|G, G|T o T $\mid \mathrm{G}$ ). Por otro lado, aquellos con puntajes más cercanos a la media del puntaje AUDIT de la muestra presentan genotipos normales o de riesgo $(\mathrm{T} \mid \mathrm{T})$ de forma aleatoria. 
que contenía la distribución de los individuos según puntaje AUDIT (Figura 4) le fue sobrepuesto como variables suplementarias los genotipos de protección del marcador $A D H 1 B^{\star} 2$ (Figura 5) y los genotipos de riesgo (ADH4, SNP6) (Figura 6).

El MCA del genotipo protector $A D H 1 B^{\star} 2$ muestra una tendencia, donde aquellos individuos que presentan un patrón de "consumo perjudicial o de dependencia" poseen un genotipo normal. Por otro lado, el genotipo protector se posiciona en el centro de la distribución, correspondiendo a las categorías de "consumo de riesgo" y "consumo sin riesgo".

El MCA de genotipo riesgoso no mostró ninguna tendencia como en el caso anterior. Los individuos con mayor puntaje AUDIT ubicados en el cuadrante I y IV presentan tanto genotipos normales como riesgosos. Existieron algunas excepciones (7 individuos) donde se encontró congruencia con respecto al patrón de consumo de alcohol y su genotipo.

Para ambos marcadores, no existieron diferencias en el porcentaje de genotipos según categoría de consumo de alcohol, así como tampoco en los promedios de puntaje AUDIT para individuos con genotipos normal o protector (Mann-Whitney, $\mathrm{W}$ $\left.=1461,5, \mathrm{p}_{\mathrm{W}}=0,3822\right)$ y normal o riesgoso $(\mathrm{W}=$ 3827,5, p-value $=0,2605$ ).

Finalmente, para contrastar la hipótesis de esta investigación se llevó a cabo un modelo de regresión lineal múltiple. Se consideró como variable dependiente el puntaje AUDIT y como independientes las variables sexo, edad, genotipo protector de $A D H 1 B 2^{\star} 2$ y genotipo de riesgo de ADH4 SNP6. El modelo no logró ser validado, con un poder de predicción y explicativo relativamente bajo $\left(\mathrm{R}^{2}\right.$ ajustado $\left.=-0,0007977\right)$ no siendo estadísticamente significativo $\left(\mathrm{p}_{\mathrm{RL}}=0,4497\right)$.

\section{Discusión}

La intención de este trabajo no es identificar las variables predictivas de esta conducta de consumo de alcohol, sino simplemente poner a prueba la hipótesis acerca de la linealidad causal entre genotipos determinantes de la metabolización del alcohol y patrón de consumo. Este modelo, corrientemente sugerido en la literatura ${ }^{11}$, no había sido directamente contrastado. Aquí refutamos la anterior hipótesis. Es decir, parafraseando el texto del genetista de poblaciones R. Lewontin (1984), "no está en nuestros genes"... o al menos no para el conjunto ADH-ALDH. Por otra parte, ofrecemos aquí un enfoque metodológico original, centrado en análisis estadístico multivariado sobre genotipos y conducta.

Respecto a los patrones de consumo, factores socioculturales como nivel socioeconómico, nivel educacional, zona de residencia podrían contribuir a su modulación ${ }^{16}$. Así, las proyecciones de los resultados de este estudio deben considerar la naturaleza de esta muestra poblacional ${ }^{29}$.

Por otro lado, si bien el puntaje AUDIT es un dato manejable para su aplicación extensiva en distintas poblaciones, la categorización de esta variable continua según la escala de la OMS y Chile puede generar clasificaciones bastante diferentes, las cuales toman relevancia a la hora de detectar individuos con consumo de alcohol problemático.

Respecto a las frecuencias génicas para las variantes protectoras de $A D H 1 B^{\star} 2$ y $A L D H 2^{\star} 2$, estas fueron relativamente bajas en la muestra de esta investigación en relación al resto de América ${ }^{28}$. Esta situación ha sido explicada por el surgimiento de estos alelos post-poblamiento americano, asociada a la agricultura o enfermedades endémicas de esta región ${ }^{6,7}$. Su baja representación constituye una limitación estadística para analizar la asociación con los variados patrones de consumo de los participantes.

Existe un alto y similar porcentaje de la variante de riesgo de $A D H 4$ (SNP6) entre Chile y el resto de América, probablemente como consecuencia del efecto fundador durante el poblamiento del continente. No obstante, recientemente se estableció que su relación con la tolerancia al alcohol no es sólo genética sino también epigenética ${ }^{30}$.

Cabe destacar que situaciones como la tolerancia funcional de los individuos ante el consumo continuo de alcohol ${ }^{31}$, como otros genes relacionados con vías neuronales ${ }^{32}$ y del gusto ${ }^{33}$, pero principalmente variables sociales y psicológicas propias de cada individuo ${ }^{34}$, sin duda son parte de un modelo de asociación entre patrones de consumo de alcohol y variables genéticas y culturales más complejo, cuyas interacciones aún no se conocen.

Finalmente, esta investigación permitió evaluar y generar un reporte actualizado sobre los patrones de consumo en estudiantes universitarios. Por otro 
lado, el análisis genético de las variantes asociadas a la tolerancia e intolerancia al alcohol constituyen la primera aproximación sobre la distribución de estos genotipos en población mestiza chilena, para el alelo de riesgo " $\mathrm{T}$ " del gen $A D H 4$ en relación a Europa, Asia y el resto de América. De esta manera, queda abierta la interrogante respecto al efecto de la historia evolutiva de la población chilena y América sobre las frecuencias genotípicas de este gen, así como corroborar la alta frecuencia de este alelo, predicha para poblaciones amerindias.

Agradecimientos: Los autores agradecen a los participantes de este estudio. A Tomás González y Pablo Varas por la recolección de muestras. Al Profesor Mauricio Moraga, Ricardo Verdugo, Michelle de Saint Pierre, Sebastián Krapivka, Nicolás Montalva y Tomás González por sus observaciones y pertinentes acotaciones durante el desarrollo de esta investigación. Finalmente a Vid-Enlace 70219.

\section{Referencias}

1. Edenberg H, Xuei X, Chen H-J, Tian H, Wetherill LF, Dick DM, et al. Association of alcohol dehydrogenase genes with alcohol dependence: a comprehensive analysis. Hum Mol Genet 2006; 15 (9): 1539-49.

2. Ehlers CL, Spence JP, Wall TL, Gidler DA, Carr LG. Association of ALDH1 Promoter Polymorphisms With Alcohol-Related Phenotypes in Southwest California Indians. Alcohol Clin Exp Res 2004; 28 (10): 1481-6.

3. Luo X, Kranzler H, Zuo L, Wang S, Schork N, Gelernter J. ADH4 Gene Variation is Associated with Alcohol Dependence and Dug Dependence in European Americans: Results from HWD Test and Case-Control Association Studies. Neuropsychopharmacology 2006; 31 (5): 108595.

4. Luo X, Kranzler H, Zuo L, Wang S, Schork N, Gelernter J. Diplotype Trend Regression Analysis of the ADH Gene Cluster and the ALDH2 Gene: Multiple Significant Associations with Alcohol Dependence. Am J Hum Genet 2006; 78 (6): 973-87. Epub 2006 Apr 11.

5. Mulligan C, Robin R, Osier M, Sambuughin N, Goldfarb $\mathrm{L}$, Kittles R, et al. Allelic variation at alcohol metabolism genes (ADH1B, ADH1C, ADLH2) and alcohol dependence in an American Indian population. Hum Genet 2003; 113 (4): 325-36. Epub 2003 Jul 12.

6. Peng Y, Shi H, Qi X, Xiao C, Zhong H, Ma R, Su B. The ADH1B Arg47His polymorphism in east Asian populations and expansion of rice domestication in history.
BMC Evolutionary Biology 2010; 10: 15.

7. Oota H, Pakstis A, Bonne-Tamir B, Goldman D, Grigorenko E, Kajuna $S$, et al. The evolution and population genetics of the ALDH2 locus: random genetic drift, selection, and low levels of recombination. Ann Hum Genet 2004; 68 (Pt 2): 93-109.

8. Gunzerath L, Hewitt BC, Li T-K, Warren KR. Alcohol research: past, present and future. Ann N Y Acad Sci 2011; 1216: 1-23.

9. Kimura M, Higuchi S. Genetics of Alcohol Dependence. Psychiatry and Clinical Neurosciences 2011; 65 (3): 21325.

10. Park CB, Kim JW, Cheong HS, Lee LH, Seo CH, Kang $\mathrm{T}-\mathrm{C}$, et al. Extended genetic effects of ADH cluster genes on the risk of alcohol dependence: from GWAS to replication. Hum Genet 2013; 132 (6): 657-68.

11. Starkman BG, Sakharkar Ph.D., AJ, Pandey PS. Epigenetics-Beyond the Genome in Alcoholism. Alcohol Research: Current Reviews 2012; 34 (3): 293-302.

12. Wang S, Ray N, Rojas W, Parra MV, Bedoya G, Gallo C, et al. Geographic Patterns of Genome Admixture in Latin. PLoS Genetics 2008; 8: 1-9.

13. Organización Mundial de la Salud. Global status report on alcohol and health. WHO Press. 2012. Disponible en http://www.who.int/ (Consultado el 12 de diciembre de 2013).

14. Observatorio Chileno de Drogas. Caracterización de los niveles de consumo de alcohol en Chile. Servicio Nacional para la Prevención y Rehabilitación del Consumo de Drogas y Alcohol, SENDA. 2004. Boletin $\mathrm{N}^{\circ}$ 4. Disponible en http://www.senda.gob.cl/ observatorio/boletines/boletin04/ (Consultado el 24 de noviembre de 2013).

15. Organización Mundial de la Salud. (Global status report on alcohol and health. WHO Press. 2014. Disponible en http://www.who.int/ (Consultado el 20 de mayo de 2014).

16. Babor TF, Higgins-Biddle JC, Saunders JB, Monteiro MG. AUDIT: Cuestionario de Identificación de Transtornos debidos al Consumo de Alcohol. 2001. Disponible en http://www.who.int/ (Consultado el 9 de noviembre de 2013).

17. Alvarado ME, Garmendia ML, Acuña G, Santis R, Arteaga O. Validez y confiabilidad de la versión chilena del Alcohol Use Disorders Identification Test (AUDIT). Rev Med Chile 2009; 137 (11): 1463-8.

18. Monteiro, Maristela G. Alcohol y salud pública en las Américas: un caso para la acción. Organizacion Panamericana de la Salud. 2007. Disponible en http://www. who.int/ (Consultado el 9 de noviembre de 2013).

19. Quinque D, Kittler R, Kayser M, Stoneking M, Nasidze. 
Evaluation of saliva as a source of human DNA for population and association studies. Anal Biochem 2006; 353 (2): 272-7.

20. National Institutes of Health de Estados Unidos and Nucleotide Sequence Database Collaboration. Base de datos GenBank. (1982). Disponible en http://www.ncbi. nlm.nih.gov/genbank/ (Consultado el 14 de octubre de 2011).

21. Rozen S, Skaletsky H. Primer3 on the WWW for general users and for biologist programmers. In: Krawetz S, Misener S (eds) Bioinformatics Methods and Protocols. Methods Mol Biol 2000; 132: 365-86.

22. Vincze T, Posfai J, Roberts RJ. NEBcutter: a program to cleave DNA with restriction enzymes. Nucleic Acids Res 2003; 31 (13): 3688-91.

23. Tamura K, Peterson D, Peterson N, Stecher G, Nei M, Kumar S. MEGA5: Molecular Evolutionary Genetics Analysis using Maximum Likelihood, Evolutionary Distance, and Maximum Parsimony Methods. Mol Biol Evol 2011; 28 (10): 2731-9.

24. R Core Team. R: A language and environment for statistical computing. R Foundation for Statistical Computing, Vienna, Austria 2004. Disponible en http:// www.R-project.org/ (Consultado el 20 de abril de 2012).

25. R Studio. (2014). RStudio: Integrated development environment for R.2014. Disponible en http://www.R-project.org/ (Consultado el 20 de abril de 2012).

26. Wickham H. Ggplot2: elegant graphics for data analysis. 2009. Springer New York.
27. Excoffier L, Lischer HL. Arlequin suite ver 3.5: A new series of programs to perform population genetics analyses under Linux and Windows. Mol Ecol Resour 2010; 10 (3): 564-7.

28. Patterson K. 1000 GENOMES: A World of Variation. Circulation Research 2011; 108: 534-6.

29. Federación de Estudiantes de la Universidad de Chile (FECH). Encuesta de Caracterización de Estudiantes Universidad de Chile 2011. Centro de Estudios Federación de Estudiantes de la Universidad de Chile (CEFECH) y Ministerio de Educación. 2011.

30. Zhang H, Wang F, Kranzler H, Yang C, Xu H, Wang Z, Gelernter J. Identification of methylation quantitative trait loci (mQTLs) influencing promoter DNA methylation of alcohol dependence risk genes. Hum Genet 2014; 133 (9): 1093-104.

31. Wallace M, Newton P, Oyasu M, McMahon T, Wen-Hai C, Connolly J, Messing R. Acute Functional Tolerance to Ethanol Mediated by Protein Kinase $C_{\varepsilon}$. Neuropsychopharmacology 2007; 32: 127-36.

32. Dick DM, Agrawal A. The Genetics of Alcohol and Other Drug Dependence. Alcohol Research \& Health 2008; 31 (2): 111-8.

33. Live Sciences: Is Booze Tasty, or Bitter? Your Genes Decide. 2014. Disponible en http://www.livescience.com/ (Consultado el 1 de octubre de 2014).

34. White H, Jackson K. Social and Psychological Influences on Emerging Adult Drinking Behavior. Alcohol Research \& Health 2004/2005, Vol. 28 (4): 182-91. 